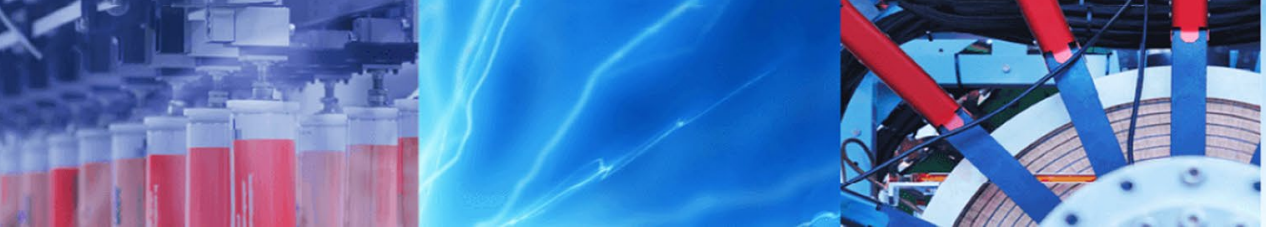

Research Article

\title{
Synthesis and mass spectrometry of some arylidene-hydrazinyl-thiazolines and their 2-arylidene- $N$-(3-chlorophenyl)hydrazinecarbothioamide precursors
}

\author{
El Sayed Ramadan ${ }^{1}$ (D)
}

() Springer Nature Switzerland AG 2019

\begin{abstract}
$\mathrm{N}$-(3-Chlorophenyl)hydrazinecarbothioamide (2) was prepared by reacting 1-chloro-3-isothiocyanatobenzene (1) with hydrazine hydrate in 2-propanol, whose ${ }^{1} \mathrm{H}$-NMR spectrum showed its existence in a Zwitter ionic betain form. A number of 2-arylidene- $N$-(3-chlorophenyl)hydrazinecarbothioamides (3a-e) were synthesized by the condensation of $\mathbf{2}$ with aromatic aldehydes in absolute ethanol and in the presence of a catalytic amount of glacial acetic acid. They were reacted with 2-bromo-1-(4-bromophenyl)ethanone (4) in 2-propanol/DMF in the presence of anhydrous potassium carbonate to produce the respective 2-(arylidenehydrazono)-4-(4-bromophenyl)-3-(3-chlorophenyl)-2,3-dihydrothiazoles (5a-e) in excellent yields. The synthesized compounds were characterized by spectroscopic methods as well as mass spectrometry. The molecular modeling and atomic charges of heteroatoms in all compounds were performed using Chem3D Pro 12.0.2 software and provided an excellent tool to investigate the fragmentation pathways. The nitrogen rule was also found to be good predictors of molecular ions and their fragmentation patterns.
\end{abstract}

Keywords Hydrazones · Nitrogen rule · Mass spectrometry · Thiazolines · Thiosemicarbazones

\section{Introduction}

Thiosemicarbazones and their metal complexes are compounds of considerable interest because of their important chemical properties and beneficial biological activities. In the last decades, there has been a growing attention towards thiosemicarbazones related to their wide range of biological properties specifically as antituberculosis, antifungal, antiviral, antimicrobial, antimalarial, antiprotozoal, antitumor and anticancer agents [1-4]. Several synthetic thiosemicarbazone derivatives already exist in the market such as triapine, marboran and thioacetazone (Fig. 1). Triapine is a potent inhibitor of ribonucleotide reductase activity with broad spectrum antitumor activity [5]. Marboran is a good antiviral agent having activity against vaccinia and cowpox virus infections [6]. Thioacetazone is one of the oldest and cheapest antibiotics used as a second line drug for tuberculosis treatment [7]. Aldehyde thiosemicarbazones are also appropriate substrates for the preparation of five or six membered heterocyclic rings that contain two heteroatoms such on treating them with oxidizing or other cyclizing agents [8-22]. In this context, nitrogen and sulfur-containing 4-thiazolines (2,3-dihydrothiazoles) and their derivatives are considered as important classes of heterocyclic compounds due to their frequent appearance in natural products and medicinal drugs [23-25]. Also, a large number of synthetic 4-thiazolines are known to exhibit a wide range of biological activities including

Electronic supplementary material The online version of this article (https://doi.org/10.1007/s42452-019-0480-x) contains supplementary material, which is available to authorized users.

El Sayed Ramadan, elsayedramadan2008@yahoo.com | 'Department of Chemistry, Faculty of Science, Alexandria University, Alexandria 21524, Egypt.

SN Applied Sciences (2019) 1:451 | https://doi.org/10.1007/s42452-019-0480-x

Received: 13 March 2019 / Accepted: 10 April 2019 / Published online: 12 April 2019

SN Applied Sciences

A SPRINGER NATURE journal 
Fig. 1 Some marketed thiosemicarbazone drugs<smiles>NC(=S)N/N=C/c1ncccc1N</smiles>

Triapine<smiles>CN1C(=O)/C(=N\NC(N)=S)c2ccccc21</smiles>

Marboran<smiles>CC(=O)Nc1ccc(/C=N/NC(N)=S)cc1</smiles>

Thioacetazone herbicidal, antibacterial, antifungal, ant-inflammatory, anticholinesterase, antitumor, and selective anti-proliferative effects [26-32].

Recently, we reported the synthesis of 2-arylidene- $\mathrm{N}$-(3chlorophenyl)hydrazinecarbothioamides along with their conversion to the respective 2-hydrazono-2,3-dihydrothiazoles [33]. Continuing our interest in the synthesis of bioactive arylidene-hydrazinyl-thiazoles [34], we report herein their modified synthesis by reacting 2-arylidene- $\mathrm{N}$-(3-chlorophenyl)hydrazinecarbothioamides with 2-bromo-1-(4bromophenyl)ethanone in 2-propanol/DMF and in the presence of anhydrous potassium carbonate as a scavenger of the liberated hydrogen bromide, which thus offered ease of reaction work-up and isolation of products in a pure state. The mass spectrometry and plausible electron impact pathways of all the synthesized 4-thiazolines and their respective thiosemicarbazones were also studied.

\section{Experimental}

\subsection{Materials}

Starting chemicals and reagents used in this study were purchased from Sigma-Aldrich. Thin layer chromatography (TLC) was performed on $0.25 \mathrm{~mm}$ aluminum silica gel plates $\left(60 \mathrm{~F}_{254}\right.$, Fluka) using ethyl acetate/petroleum ether (2:3 volume ratio) and ethyl acetate/methylene chloride (4:1 volume ratio) as eluents; the spots were detected by using UV light absorption.

\subsection{Apparatus and instruments}

Melting points were determined with a Mel-Temp apparatus and are uncorrected. The mass spectrometry was recorded on a Shimadzu Qp-2010 Plus GC-MS mass spectrometer with ion source temperature $250{ }^{\circ} \mathrm{C}$, using the direct inlet probe technique at an ionizing voltage of $70 \mathrm{eV}$. FT-IR spectra were measured on a Bruker Tensor $37 \mathrm{FT}-\mathrm{IR}$ spectrometer. ${ }^{1} \mathrm{H}$-NMR were recorded on a JEOL JNM ECA-500 spectrometer at $500 \mathrm{MHz}$. Chemical shifts $(\delta)$ are given in parts per million (ppm) relative to TMS as an internal standard and coupling constants $(J)$ are reported in hertz.

\subsection{Synthesis of $\mathrm{N}$-(3-chlorophenyl)hydrazinecar- bothioamide (2)}

To a stirred solution of hydrazine hydrate $(100 \%, 5.0 \mathrm{~mL}$, $0.10 \mathrm{~mol}$ ) in $20 \mathrm{~mL}$ of 2-propanol, 1-chloro-3-isothiocyanatobenzene $(\mathbf{1})(13.10 \mathrm{~mL}, 0.10 \mathrm{~mol})$ was added dropwise at room temperature with occasional stirring during $10 \mathrm{~min}$. The precipitate was formed immediately, and stirring was continued for further $20 \mathrm{~min}$. The mixture was filtered, and the precipitate was recrystallized three times from absolute ethanol as white crystals. Yield: $85 \%$ $\left(1.222\right.$ g); m.p.: $119-120{ }^{\circ} \mathrm{C}$; Lit. [35] m.p.: $119-120^{\circ} \mathrm{C}$; $\mathrm{R}_{\mathrm{f}}=0.68$ (ethyl acetate/petroleum ether, $2: 3 \mathrm{v} / \mathrm{v}$ ); $\mathrm{IR}(\mathrm{KBr})$ : $v=3331.96,3279.59,3195.80\left(\mathrm{NH}_{1} \mathrm{NH}_{2}\right), 2935.39(\mathrm{C}-\mathrm{H})$, $1630.62,1590.72,1522.94(\mathrm{C}=\mathrm{C}, \mathrm{C}=\mathrm{N}), 1487.31(\mathrm{~N}-\mathrm{CS}-\mathrm{N})$, $1253.81(\mathrm{C}=\mathrm{S}), 966.14(\mathrm{C}-\mathrm{Cl}) \mathrm{cm}^{-1} ;{ }^{1} \mathrm{H}-\mathrm{NMR}(500 \mathrm{MHz}$, DMSO- $\left.d_{6}\right): \delta=4.85$ (broad s, $2 \mathrm{H}, \mathrm{D}_{2} \mathrm{O}$-exchangeable, $\mathrm{NH}_{2}$ ), $7.09(\mathrm{~d}, J=6.9 \mathrm{~Hz}, 1 \mathrm{H}, \mathrm{Ar}-\mathrm{H}), 7.26(\mathrm{t}, J=7.65 \mathrm{~Hz}, 1 \mathrm{H}, \mathrm{Ar}-\mathrm{H})$, $7.53(\mathrm{~d}, J=6.9 \mathrm{~Hz}, 1 \mathrm{H}, \mathrm{Ar}-\mathrm{H}), 7.96(\mathrm{~s}, 1 \mathrm{H}, \mathrm{Ar}-\mathrm{H}), 9.26(\mathrm{~s}, 2 \mathrm{H}$, $\mathrm{D}_{2} \mathrm{O}$-exchangeable, $\mathrm{NH}_{2}$ ) ppm.

\subsection{Synthesis of 2-arylidene- $\mathrm{N}$-(3-chlorophenyl) hydrazinecarbothioamides (3a-e)}

$\mathrm{N}$-(3-Chlorophenyl)hydrazinecarbothioamide (2) $(2.01 \mathrm{~g}$, $10 \mathrm{mmol})$ in absolute ethanol $(30 \mathrm{~mL})$ and glacial acetic acid $(1.0 \mathrm{~mL})$ was warmed on a steam bath to a clear solution and added to an aromatic aldehyde $(10 \mathrm{mmol})$ in absolute ethanol $(20 \mathrm{~mL})$. The reaction mixture was heated under reflux for $1 \mathrm{~h}$ and the product that separated out on cooling was filtered, washed with a little ethanol and dried. It was recrystallized from ethanol to give the title compounds.

\subsubsection{2-Benzylidene- $\mathrm{N}$-(3-chlorophenyl)hydrazinecarboth- ioamide (3a)}

White plates, $80 \%$ yield, m. p.: $143-146^{\circ} \mathrm{C}$; Lit. [33] m.p.: 144-146 ${ }^{\circ} \mathrm{C} ; R_{\mathrm{f}}=0.64$ (ethyl acetate/petroleum ether, $2: 3$ $\mathrm{v} / \mathrm{v}) ;{ }^{1} \mathrm{H}-\mathrm{NMR}\left(500 \mathrm{MHz}\right.$, DMSO- $\left.d_{6}\right): \delta=7.22,7.23(\mathrm{dd}, J=7.6$, 
$1.5 \mathrm{~Hz}, 1 \mathrm{H}, \mathrm{Ar}-\mathrm{H}), 7.36(\mathrm{t}, J=8.4 \mathrm{~Hz}, 1 \mathrm{H}, \mathrm{Ar}-\mathrm{H}), 7.39-7.41(\mathrm{~m}$, $3 \mathrm{H}, \mathrm{Ar}-\mathrm{H}), 7.56(\mathrm{~d}, J=8.4 \mathrm{~Hz}, 1 \mathrm{H}, \mathrm{Ar}-\mathrm{H}), 7.72(\mathrm{t}, J=1.5 \mathrm{~Hz}$, $1 \mathrm{H}, \mathrm{Ar}-\mathrm{H}), 7.86,7.87$ (dd, J=7.6, $2.3 \mathrm{~Hz}, 2 \mathrm{H}, \mathrm{Ar}-\mathrm{H}), 8.13(\mathrm{~s}$, $1 \mathrm{H}, \mathrm{CH}=\mathrm{N}), 10.15$ (s, 1H, $\mathrm{D}_{2} \mathrm{O}$-exchangeable, NH-Ar), 11.93 (s, $1 \mathrm{H}, \mathrm{D}_{2} \mathrm{O}$-exchangeable, NH-CS) ppm; El-MS m/z (\% rel. abund.): 291.05 ([M+2 $\left.]^{+}, 16.16\right), 289.05\left([\mathrm{M}]^{+}, 43.64\right), 187.00$ (24.45), 185.05 (67.06), 171.00 (2.74), 169.05 (4.86), 162.05 (9.62), 129.10 (31.09), 127.10 (100.00), 119.15 (51.75), 113.10 (7.80), 111.10 (23.54), 104.15 (24.96), 92.10 (15.18), 77.10 (61.31), 76.15 (12.12), 75.10 (21.53).

\subsubsection{N-(3-Chlorophenyl)-2-(4-nitrobenzylidene)hydrazine- carbothioamide (3b)}

Yellow crystals, $88 \%$ yield, m. p.: $228-230{ }^{\circ} \mathrm{C}$; Lit. [33] m.p.: $228-230{ }^{\circ} \mathrm{C} ; \mathrm{R}_{\mathrm{f}}=0.66$ (ethyl acetate/petroleum ether, $2: 3 \mathrm{v} / \mathrm{v}) ;{ }^{1} \mathrm{H}-\mathrm{NMR}\left(500 \mathrm{MHz}\right.$, DMSO- $\left.d_{6}\right): \delta=7.25,7.26$ (dd, $J=8.4,1.55 \mathrm{~Hz}, 1 \mathrm{H}, \mathrm{Ar}-\mathrm{H}), 7.38(\mathrm{t}, J=8.4 \mathrm{~Hz}, 1 \mathrm{H}, \mathrm{Ar}-\mathrm{H}), 7.53$ $(\mathrm{d}, J=8.4 \mathrm{~Hz}, 1 \mathrm{H}, \mathrm{Ar}-\mathrm{H}), 7.68(\mathrm{t}, J=1.55 \mathrm{~Hz}, 1 \mathrm{H}, \mathrm{Ar}-\mathrm{H}), 8.15(\mathrm{~d}$, $J=9.15 \mathrm{~Hz}, 2 \mathrm{H}, \mathrm{Ar}-\mathrm{H}), 8.21(\mathrm{~s}, 1 \mathrm{H}, \mathrm{CH}=\mathrm{N}), 8.23(\mathrm{~d}, J=9.15 \mathrm{~Hz}$, $2 \mathrm{H}, \mathrm{Ar}-\mathrm{H}), 10.34$ (s, 1H, $\mathrm{D}_{2} \mathrm{O}$-exchangeable, $\mathrm{NH}-\mathrm{Ar}$ ), 12.19 (s, 1H, D 2 -exchangeable, NH-CS) ppm; El-MS m/z (\% rel. abund.): $336.05\left([\mathrm{M}+2]^{+}, 6.08\right), 334.00\left([\mathrm{M}]^{+}, 20.89\right), 207.00$ (19.49), 187.00 (10.50), 185.05 (28.72), 171.00 (11.40), 169.00 (28.26), 164.10 (4.86), 149.10 (14.52), 129.10 (31.44), 127.10 (100.00), 122.10 (4.83), 113.10 (9.94), 111.10 (29.85), 92.10 (19.35), 76.10 (39.91), 75.10 (37.67).

\subsubsection{N-(3-Chlorophenyl)-2-(4-methoxybenzylidene)hydra- zinecarbothioamide (3c)}

Pale yellow plates, $83 \%$ yield, m. p.: $166-168{ }^{\circ} \mathrm{C}$; Lit. [33] m.p.: $166-168^{\circ} \mathrm{C} ; \mathrm{R}_{\mathrm{f}}=0.60$ (ethyl acetate/petroleum ether, $2: 3 \mathrm{v} / \mathrm{v}) ;{ }^{1} \mathrm{H}-\mathrm{NMR}\left(500 \mathrm{MHz}, \mathrm{DMSO}-d_{6}\right): \delta=3.77(\mathrm{~s}, 3 \mathrm{H}$, $\left.\mathrm{OCH}_{3}\right), 6.94(\mathrm{~d}, J=9.15 \mathrm{~Hz}, 2 \mathrm{H}, \mathrm{Ar}-\mathrm{H}), 7.21,7.22$ (dd, $J=9.15$, $1.55 \mathrm{~Hz}, 1 \mathrm{H}, \operatorname{Ar}-\mathrm{H}), 7.35(\mathrm{t}, J=8.4 \mathrm{~Hz}, 1 \mathrm{H}, \operatorname{Ar}-\mathrm{H}), 7.56(\mathrm{~d}$, $J=8.4 \mathrm{~Hz}, 1 \mathrm{H}, \mathrm{Ar}-\mathrm{H}), 7.73(\mathrm{~s}, 1 \mathrm{H}, \mathrm{Ar}-\mathrm{H}), 7.81(\mathrm{~d}, J=8.4 \mathrm{~Hz}, 2 \mathrm{H}$, Ar-H), $8.08(\mathrm{~s}, 1 \mathrm{H}, \mathrm{CH}=\mathrm{N}), 10.08\left(\mathrm{~s}, 1 \mathrm{H}, \mathrm{D}_{2} \mathrm{O}\right.$-exchangeable, $\mathrm{NH}-\mathrm{Ar}$ ), 11.82 (s, 1H, D $\mathrm{O}$-exchangeable, NH-CS) ppm; El-MS $m / z$ (\% rel. abund.): $321.05\left([\mathrm{M}+2]^{+}, 8.09\right), 319.05$ $\left([\mathrm{M}]^{+}, 24.94\right), 192.05$ (53.63), 187.00 (7.67), 185.00 (21.04), 171.00 (4.30), 169.00 (10.95), 149.10 (15.07), 134.15 (54.04), 129.10 (31.03), 127.10 (100.00), 113.10 (5.47), 111.10 (16.53), 107.15 (21.08), 92.10 (40.92), 76.10 (13.68), 75.10 (18.76).

\subsubsection{2-(2-Chlorobenzylidene)-N-(3-chlorophenyl)hydrazi- necarbothioamide (3d)}

Yellow crystals, $85 \%$ yield, m. p.: $192-194{ }^{\circ} \mathrm{C}$; Lit. [33] m.p.: $192-194{ }^{\circ} \mathrm{C} ; \mathrm{R}_{\mathrm{f}}=0.56$ (ethyl acetate/petroleum ether, 2:3 v/v); ${ }^{1} \mathrm{H}-\mathrm{NMR}\left(500 \mathrm{MHz}, \mathrm{DMSO}-d_{6}\right): \delta=7.23(\mathrm{~d}$, $J=7.65 \mathrm{~Hz}, 1 \mathrm{H}, \operatorname{Ar}-\mathrm{H}), 7.35-7.41(\mathrm{~m}, 3 \mathrm{H}, \operatorname{Ar}-\mathrm{H}), 7.46(\mathrm{~d}$,
$J=7.65 \mathrm{~Hz}, 1 \mathrm{H}, \mathrm{Ar}-\mathrm{H}), 7.54(\mathrm{~d}, J=8.4 \mathrm{~Hz}, 1 \mathrm{H}, \mathrm{Ar}-\mathrm{H}), 7.70$ (s, $1 \mathrm{H}, \mathrm{Ar}-\mathrm{H}), 8.41(\mathrm{~d}, J=6.9 \mathrm{~Hz}, 1 \mathrm{H}, \mathrm{Ar}-\mathrm{H}), 8.57(\mathrm{~s}, 1 \mathrm{H}, \mathrm{CH}=\mathrm{N})$, 10.23 (s, $1 \mathrm{H}, \mathrm{D}_{2} \mathrm{O}$-exchangeable, NH-Ar), $12.10(\mathrm{~s}, 1 \mathrm{H}$, $\mathrm{D}_{2} \mathrm{O}$-exchangeable, NH-CS) ppm; El-MS $\mathrm{m} / \mathrm{z}$ (\% rel. abund.): $326.95\left([\mathrm{M}+4]^{+}, 5.38\right), 325.00\left([\mathrm{M}+2]^{+}, 23.09\right), 323.00\left([\mathrm{M}]^{+}\right.$, 32.08), 198.00 (1.56), 196.00 (3.68), 187.00 (26.32), 185.05 (71.13), 171.05 (4.24), 169.05 (9.26), 155.05 (7.60), 153.05 (21.82), 140.10 (9.03), 138.10 (16.78), 129.10 (30.99), 127.10 (100.00), 113.10 (17.22), 111.10 (50.14), 92.10 (17.12), 76.05 (11.24), 75.05 (36.07).

\subsection{5 (E/Z)-N-(3-Chlorophenyl)-2-(2-hydroxybenzylidene) hydrazinecarbothioamide (3e)}

White plates, $82 \%$ yield, m. p.: $176-178{ }^{\circ} \mathrm{C}$; Lit. [33] m.p.: $176-178{ }^{\circ} \mathrm{C} ; \mathrm{R}_{\mathrm{f}}=0.58$ (ethyl acetate/petroleum ether, $2: 3$ $\mathrm{v} / \mathrm{v}) ;{ }^{1} \mathrm{H}-\mathrm{NMR}\left(500 \mathrm{MHz}, \mathrm{DMSO}-d_{6}\right): \delta=[6.81(\mathrm{t}, J=6.85 \mathrm{~Hz}$, Ar-H), $6.84(\mathrm{~d}, J=7.65 \mathrm{~Hz}, \mathrm{Ar}-\mathrm{H}), 6.92-6.95(\mathrm{~m}, \mathrm{Ar}-\mathrm{H})$, 7.19-7.22 (m, Ar-H), 7.33-7.36 (m, Ar-H), $7.55(\mathrm{~d}, J=8.4 \mathrm{~Hz}$, Ar-H), 7.65, 7.67 (dd, J=7.65, $1.5 \mathrm{~Hz}, \mathrm{Ar}-\mathrm{H}), 7.73$ (t, $J=1.5 \mathrm{~Hz}, \mathrm{Ar}-\mathrm{H}), 8.05(\mathrm{~d}, J=6.9 \mathrm{~Hz}, \mathrm{Ar}-\mathrm{H})$, the total integral of $\mathrm{Ar}-\mathrm{H}=8 \mathrm{H}], 8.46(\mathrm{~s}, 0.71 \mathrm{H}, \mathrm{CH}=\mathrm{N}), 8.97(\mathrm{~s}, 0.29 \mathrm{H}, \mathrm{CH}=\mathrm{N})$, $9.98\left(\mathrm{~s}, 0.71 \mathrm{H}, \mathrm{D}_{2} \mathrm{O}\right.$-exchangeable, $\left.\mathrm{OH}\right), 11.10(\mathrm{~s}, 0.29 \mathrm{H}$, $\mathrm{D}_{2} \mathrm{O}$-exchangeable, $\left.\mathrm{OH}\right), 10.08\left(\mathrm{~s}, 1 \mathrm{H}, \mathrm{D}_{2} \mathrm{O}\right.$-exchangeable, $\mathrm{NH}-\mathrm{Ar}$ ), 11.86 (s, 1H, D O-exchangeable, NH-CS) ppm; El-MS $m / z$ (\% rel. abund.): $307.00\left([\mathrm{M}+2]^{+}, 19.12\right), 305.00$ $\left([\mathrm{M}]^{+}, 52.79\right), 187.00$ (15.10), 185.00 (41.28), 178.00 (16.59), 171.00 (7.52), 169.00 (18.10), 135.10 (20.73), 129.10 (31.40), 127.10 (100.00), 120.10 (33.49), 113.05 (9.36), 111.05 (27.95), 93.10 (15.96), 92.10 (18.95), 76.05 (10.93), 75.05 (25.71).

\subsection{Synthesis of 2-(arylidenehydrazono)-4-(4-brom ophenyl)-3-(3-chlorophenyl)-2,3-dihydrothia- zoles (5a-e)}

A solution of $\mathbf{3 a - e}(10 \mathrm{mmol})$ in iso-propyl alcohol $(25 \mathrm{~mL})$ and $N, N$-dimethylformamide $(2.0 \mathrm{~mL})$ was treated with the solution of compound $4(2.78 \mathrm{~g}, 10 \mathrm{mmol})$ in iso-propyl alcohol $(25 \mathrm{~mL})$ and anhydrous potassium carbonate $(1.38 \mathrm{~g}, 10 \mathrm{mmol})$. The reaction mixture was heated under reflux for $2 \mathrm{~h}$ and left to cool to room temperature and the reaction progress was monitored by TLC using ethyl acetate/methylene chloride (4:1 volume ratio). The separated product was filtered, washed twice with saturated solution of $\mathrm{NaHCO}_{3}(30 \mathrm{~mL})$ and recrystallized from a mixture of EtOH and DMF.

\subsubsection{2-(Benzylidenehydrazono)-4-(4-bromophenyl)-3-(3- chlorophenyl)-2,3-dihydrothiazole (5a)}

Yellow crystals, $84 \%$ yield, m. p.: $232-234{ }^{\circ} \mathrm{C}$; Lit. [33] m.p.: $232-234{ }^{\circ} \mathrm{C} ; \mathrm{R}_{\mathrm{f}}=0.50$ (ethyl acetate/methylene chloride, 4:1 
$\mathrm{v} / \mathrm{v}) ;{ }^{1} \mathrm{H}-\mathrm{NMR}\left(500 \mathrm{MHz}\right.$, DMSO- $\left.d_{6}\right): \delta=6.69\left(\mathrm{~s}, 1 \mathrm{H}, \mathrm{C}_{5}-\mathrm{H}\right.$ of thiazoline ring), $7.11(\mathrm{~d}, J=8.4 \mathrm{~Hz}, 2 \mathrm{H}, \mathrm{Ar}-\mathrm{H}), 7.16,7.17$ (tt, $J=8.4,2.3 \mathrm{~Hz}, 1 \mathrm{H}, \mathrm{Ar}-\mathrm{H}), 7.32-7.39(\mathrm{~m}, 5 \mathrm{H}, \mathrm{Ar}-\mathrm{H}), 7.44(\mathrm{~d}$, $J=8.4 \mathrm{~Hz}, 2 \mathrm{H}, \mathrm{Ar}-\mathrm{H}), 7.51(\mathrm{~s}, 1 \mathrm{H}, \mathrm{Ar}-\mathrm{H}), 7.65(\mathrm{~d}, J=6.9 \mathrm{~Hz}, 2 \mathrm{H}$, Ar-H), 8.17 (s, 1H, CH=N) ppm; El-MS $\mathrm{m} / \mathrm{z}$ (\% rel. abund.): $470.95\left([\mathrm{M}+4]^{+}, 30.98\right), 468.95\left([\mathrm{M}+2]^{+}, 100.00\right), 467.00$ $\left([\mathrm{M}]^{+}, 72.58\right), 353.95$ (20.53), 351.95 (70.98), 349.95 (53.28), 214.00 (12.31), 211.95 (7.94), 171.00 (4.96), 169.00 (12.73), 134.10 (18.84), 113.10 (11.65), 111.10 (36.06), 101.15 (20.87), 90.10 (97.38), 76.15 (14.47), 75.10 (36.53).

\subsubsection{4-(4-Bromophenyl)-3-(3-chlorophenyl)-2-[(4-nitro benzylidene)hydrazono]-2,3-dihydrothiazole (5b)}

Orange crystals, $85 \%$ yield, m. p.: $240-242^{\circ} \mathrm{C}$; Lit. [33] m.p.: $240-242^{\circ} \mathrm{C} ; \mathrm{R}_{\mathrm{f}}=0.52$ (ethyl acetate/methylene chloride, 4:1 $\mathrm{v} / \mathrm{v}) ;{ }^{1} \mathrm{H}-\mathrm{NMR}\left(500 \mathrm{MHz}\right.$, DMSO- $\left.d_{6}\right): \delta=6.79\left(\mathrm{~s}, 1 \mathrm{H}, \mathrm{C}_{5}-\mathrm{H}\right.$ of thiazoline ring), $7.12(\mathrm{~d}, J=8.4 \mathrm{~Hz}, 2 \mathrm{H}, \mathrm{Ar}-\mathrm{H}), 7.19,7.20$ (dd, $J=6.85,1.5 \mathrm{~Hz}, 1 \mathrm{H}, \mathrm{Ar}-\mathrm{H}), 7.35-7.40(\mathrm{~m}, 2 \mathrm{H}, \mathrm{Ar}-\mathrm{H}), 7.46(\mathrm{~d}$, $J=8.4 \mathrm{~Hz}, 2 \mathrm{H}, \mathrm{Ar}-\mathrm{H}), 7.54(\mathrm{~s}, 1 \mathrm{H}, \mathrm{Ar}-\mathrm{H}), 7.87(\mathrm{~d}, J=9.15 \mathrm{~Hz}$, $2 \mathrm{H}, \mathrm{Ar}-\mathrm{H}), 8.22(\mathrm{~d}, J=9.15 \mathrm{~Hz}, 2 \mathrm{H}, \mathrm{Ar}-\mathrm{H}), 8.28(\mathrm{~s}, 1 \mathrm{H}, \mathrm{CH}=\mathrm{N})$ ppm; El-MS m/z (\% rel. abund.): $515.95\left([\mathrm{M}+4]^{+}, 29.92\right)$, $514.00\left([\mathrm{M}+2]^{+}, 100.00\right), 512.00\left([\mathrm{M}]^{+}, 73.29\right), 353.95$ (11.65), 351.95 (40.33), 349.95 (35.73), 213.95 (8.27), 211.95 (7.66), 171.00 (7.61), 169.00 (18.87), 135.10 (3.83), 134.10 (17.10), 113.10 (8.47), 111.10 (26.12), 101.10 (13.37), 89.10 (47.85), 76.10 (15.56), 75.10 (24.01).

\subsubsection{4-(4-Bromophenyl)-3-(3-chlorophenyl)-2-[(4-meth- oxybenzylidene)hydrazono]-2,3-dihydrothiazole (5c)}

Pale yellow crystals, $87 \%$ yield, m. p.: $218-220^{\circ} \mathrm{C}$; Lit. [33] m.p.: $218-220^{\circ} \mathrm{C} ; \mathrm{R}_{\mathrm{f}}=0.55$ (ethyl acetate/methylene chloride, $4: 1 \mathrm{v} / \mathrm{v}) ;{ }^{1} \mathrm{H}-\mathrm{NMR}\left(500 \mathrm{MHz}\right.$, DMSO- $\left.d_{6}\right): \delta=3.75$ $\left(\mathrm{s}, 3 \mathrm{H}, \mathrm{OCH}_{3}\right), 6.65\left(\mathrm{~s}, 1 \mathrm{H}, \mathrm{C}_{5}-\mathrm{H}\right.$ of thiazoline ring), $6.93(\mathrm{~d}$, $J=8.4 \mathrm{~Hz}, 2 \mathrm{H}, \mathrm{Ar}-\mathrm{H}), 7.10(\mathrm{~d}, J=8.4 \mathrm{~Hz}, 2 \mathrm{H}, \mathrm{Ar}-\mathrm{H}), 7.14,7.15$ (dd, J=6.5, 1.5 Hz, 1H, Ar-H), 7.32-7.35 (m, 2H, Ar-H), 7.43 (d, J=8.4 Hz, 2H, Ar-H), $7.48(\mathrm{~s}, 1 \mathrm{H}, \mathrm{Ar}-\mathrm{H}), 7.59(\mathrm{~d}, J=8.4 \mathrm{~Hz}$, $2 \mathrm{H}, \mathrm{Ar}-\mathrm{H}$ ), $8.10(\mathrm{~s}, 1 \mathrm{H}, \mathrm{CH}=\mathrm{N}$ ) ppm; El-MS $\mathrm{m} / \mathrm{z}$ (\% rel. abund.): $501.00\left([\mathrm{M}+4]^{+}, 32.16\right), 499.00\left([\mathrm{M}+2]^{+}, 100.00\right)$, 497.00 ([M] $\left.]^{+}, 75.17\right), 353.90$ (20.38), 351.95 (69.76), 349.95 (54.60), 213.95 (2.81), 212.00 (2.61), 171.00 (2.27), 169.00 (5.80), 134.10 (10.42), 120.15 (71.15), 113.10 (4.99), 111.05 (14.91), 101.10 (7.72), 89.10 (14.85), 76.15 (6.91), 75.10 (11.08).

\subsubsection{4-(4-Bromophenyl)-2-[(2-chlorobenzylidene)hydrazo no]-3-(3-chlorophenyl)-2,3-dihydrothiazole (5d)}

Yellow crystals, $88 \%$ yield, m. p.: $246-248^{\circ} \mathrm{C}$; Lit. [33] m.p.: $246-248^{\circ} \mathrm{C} ; \mathrm{R}_{\mathrm{f}}=0.59$ (ethyl acetate/methylene chloride, $4: 1 \mathrm{v} / \mathrm{v}) ;{ }^{1} \mathrm{H}-\mathrm{NMR}\left(500 \mathrm{MHz}\right.$, DMSO- $\left.d_{6}\right): \delta=6.73\left(\mathrm{~s}, 1 \mathrm{H}, \mathrm{C}_{5}-\mathrm{H}\right.$ of thiazoline ring), $7.10(\mathrm{~d}, J=8.4 \mathrm{~Hz}, 2 \mathrm{H}, \mathrm{Ar}-\mathrm{H}), 7.20(\mathrm{~d}$,
$J=6.9 \mathrm{~Hz}, 1 \mathrm{H}, \mathrm{Ar}-\mathrm{H}), 7.37-7.38(\mathrm{~m}, 4 \mathrm{H}, \mathrm{Ar}-\mathrm{H}), 7.44-7.50(\mathrm{~m}$, $4 \mathrm{H}, \mathrm{Ar}-\mathrm{H}), 7.94(\mathrm{~s}, 1 \mathrm{H}, \mathrm{Ar}-\mathrm{H}), 8.33(\mathrm{~s}, 1 \mathrm{H}, \mathrm{CH}=\mathrm{N}) \mathrm{ppm}$; El-MS $\mathrm{m} / \mathrm{z}$ (\% rel. abund.): 506.90 ([M+6] $\left.]^{+}, 5.06\right), 504.90\left([\mathrm{M}+4]^{+}\right.$, 27.16), $502.90\left([\mathrm{M}+2]^{+}, 53.75\right), 500.90\left([\mathrm{M}]^{+}, 31.24\right), 353.95$ (11.49), 351.95 (38.93), 349.95 (29.67), 213.95 (9.13), 211.95 (8.70), 171.00 (5.78), 169.00 (14.43), 134.10 (17.49), 126.10 (8.33), 124.10 (23.74), 113.10 (15.39), 111.10 (47.15), 101.15 (21.67), 89.10 (100.00), 76.10 (12.92), 75.10 (52.39).

\subsubsection{2-[\{4-(4-Bromophenyl)-3-(3-chlorophenyl)thia- zol-2(3H)-ylidene)hydrazono\}methyl]phenol (5e)}

Pale yellow crystals, $81 \%$ yield, m. p.: $224-226^{\circ} \mathrm{C}$; Lit. [33] m.p.: $224-226^{\circ} \mathrm{C} ; \mathrm{R}_{\mathrm{f}}=0.51$ (ethyl acetate/methylene chloride, $4: 1 \mathrm{v} / \mathrm{v}) ;{ }^{1} \mathrm{H}-\mathrm{NMR}\left(500 \mathrm{MHz}\right.$, DMSO- $\left.d_{6}\right): \delta=6.73$ (s, $1 \mathrm{H}, \mathrm{C}_{5}-\mathrm{H}$ of thiazoline ring), $6.86(\mathrm{t}, J=6.85 \mathrm{~Hz}, 1 \mathrm{H}, \mathrm{Ar}-\mathrm{H})$, $6.88(\mathrm{~d}, J=8.4 \mathrm{~Hz}, 1 \mathrm{H}, \mathrm{Ar}-\mathrm{H}), 7.12(\mathrm{~d}, J=8.4 \mathrm{~Hz}, 2 \mathrm{H}, \mathrm{Ar}-\mathrm{H})$, 7.19-7.23 (m, 2H, Ar-H), 7.34-7.37 (m, 2H, Ar-H), 7.41, 7.43 (dd, $J=8.4,2.3 \mathrm{~Hz}, 1 \mathrm{H}, \mathrm{Ar}-\mathrm{H}), 7.44(\mathrm{~d}, J=8.4 \mathrm{~Hz}, 2 \mathrm{H}, \mathrm{Ar}-\mathrm{H})$, $7.54(\mathrm{t}, J=2.3 \mathrm{~Hz}, 1 \mathrm{H}, \mathrm{Ar}-\mathrm{H}), 8.41(\mathrm{~s}, 1 \mathrm{H}, \mathrm{CH}=\mathrm{N}), 11.02$ (brs, $1 \mathrm{H}, \mathrm{OH}) \mathrm{ppm}$; El-MS m/z (\% rel. abund.): $486.90\left([\mathrm{M}+4]^{+}\right.$, 30.97), $484.90\left([\mathrm{M}+2]^{+}, 100.00\right), 482.95\left([\mathrm{M}]^{+}, 74.68\right), 353.90$ (22.51), 351.90 (82.44), 349.90 (63.84), 213.95 (6.85), 211.90 (6.00), 171.00 (4.23), 169.00 (8.69), 134.10 (25.43), 113.05 (10.03), 111.05 (30.71), 106.10 (11.77), 101.10 (12.12), 89.10 (18.36), 76.10 (8.36), 75.10 (25.65).

\section{Results and discussion}

The starting material, $\mathrm{N}$-(3-chlorophenyl)hydrazinecarbothioamide (2) was prepared in $85 \%$ yield from 1-chloro3-isothiocyanatobenzene (1) and hydrazine hydrate in 2-propanol at room temperature as described [36, 37]. In the present work, its ${ }^{1} \mathrm{H}-\mathrm{NMR}$ in deuterated dimethyl sulfoxide differed from both reports, whereby two proton signals appeared at $\delta=4.85$ and $9.26 \mathrm{ppm}$, both are exchangeable with $\mathrm{D}_{2} \mathrm{O}$ and each is equivalent to two protons indicating the presence of two $\mathrm{NH}_{2}$ groups. Thus, compound $\mathbf{2}$ may exist in Zwitter ionic betain form (2C) resulting from the expected thioamide (2A) and imidethiol (2B) tautomeric equilibrium states (Scheme 1). In harmony with their highly polarized molecular structures, these species are important starting materials in the synthesis of a variety of heterocylic compounds containing nitrogen and sulphur atoms.

Although the presence of a chlorine atom at the meta position of phenyl ring in compound $\mathbf{2}$ was reported to decrease the net negative charge of the terminal nitrogen atom [35], the distance between chlorine and terminal nitrogen is too long in order to be significantly influenced the nucleophilic character of this nitrogen. Thus, it was condensed very smoothly with aromatic aldehydes to give the respective 


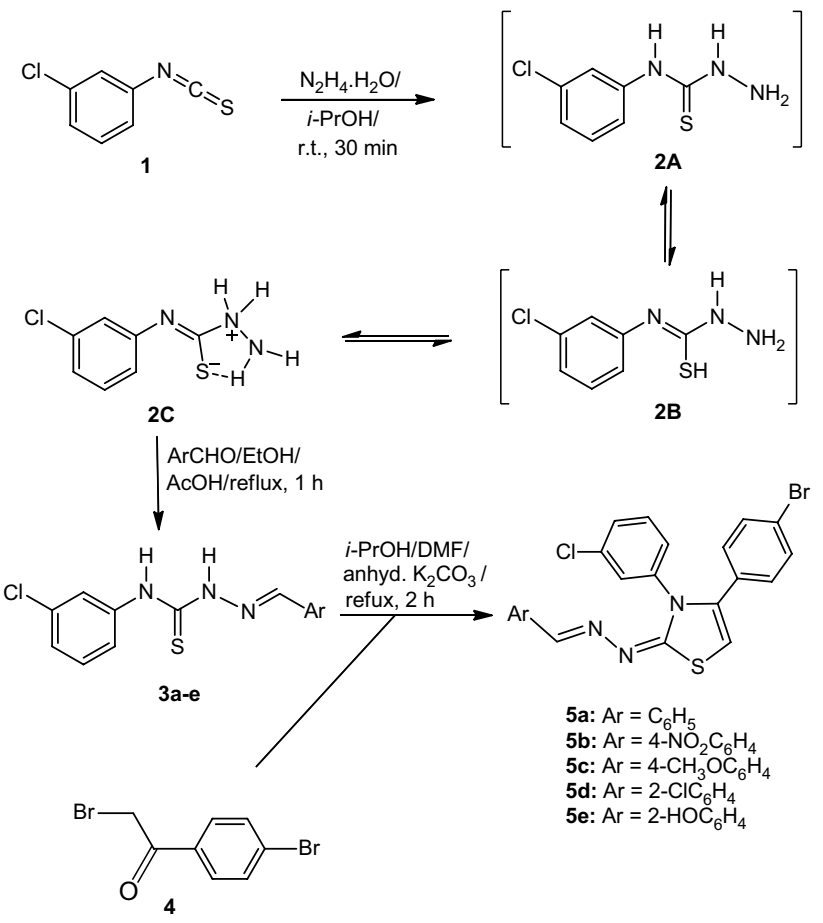

Scheme 1 Synthetic approach of compounds 2, 3a-e and 5a-e

thiosemicarbazones 3a-e in excellent yields. Compounds 3a-e were easily converted into 2 -(arylidenehydrazono)4-(4-bromophenyl)-3-(3-chlorophenyl)-2,3-dihydrothiazoles (5a-e) by a convenient Hantzsch condensation with 2-bromo-1-(4-bromophenyl)ethanone (4) in the presence of anhydrous potassium carbonate in 2-propanol and $\mathrm{N}, \mathrm{N}$ dimethylformamide as cosolvent.

Mass spectrometry (MS) is probably one of the most versatile and comprehensive analytical technique currently available in the chemists and biochemists arsenal. Today, mass spectrometry can be used to determine molecular structures to study reaction dynamics and ion chemistry. The mass spectrometry of heterocyclic compounds has been the object of considerable study, but a few reports appeared on the mass spectrometry of thiosemicarbazones and 2-hydrazono-4-thiazolines. An interest in the mass spectrometric characterization of such class of compounds is determined by using GC-MS Qp-2010 Plus gas chromatograph mass spectrometer under electron impact (EI) mode at ionizing energy of $70 \mathrm{eV}$. The concept of charge localization at specific sites in a molecule has led to mechanistic interpretations of the fragmentation of relatively simple molecules and this principle would also appear to be valid when applied to more complex structures. Thus, we report herein the results obtained in the study of correlation between fragmentation patterns in electronic impact mass spectrometry (EI-MS) and electronic charges of the heteroatoms
Table 1 Electronic charges of $\mathrm{S}_{10}, \mathrm{~N}_{7}, \mathrm{~N}_{9}$ and $\mathrm{N}_{11}$ atoms of compounds 3a-e<smiles>[X]c1ccc(/C=N/N([AlH2])C(=S)Nc2cccc(Cl)c2)cc1</smiles>

\begin{tabular}{lllllr}
\hline Compound & $\mathrm{X}$ & $\mathrm{N}_{7}$ & $\mathrm{~N}_{9}$ & \multicolumn{1}{l}{$\mathrm{S}_{10}$} & \multicolumn{1}{c}{$\mathrm{N}_{11}^{\mathrm{a}}$} \\
\hline 3a & $\mathrm{H}$ & 0.399 & 0.700 & -0.721 & -0.113 \\
3b & $4-\mathrm{NO}_{2}$ & 0.401 & 0.699 & -0.663 & 0.070 \\
3c & $4-\mathrm{OCH}_{3}$ & 0.401 & 0.709 & -0.729 & -0.110 \\
3d & $2-\mathrm{Cl}$ & 0.385 & 0.514 & -0.744 & 0.177 \\
3e & $2-\mathrm{OH}$ & 0.399 & 0.685 & -0.718 & 0.042 \\
\hline
\end{tabular}

${ }^{a}$ Numbering of atoms is related to molecular modeling structure

of some arylidene-hydrazinyl-thiazolines and their thiosemicarbazone precursors. The initial charge localization may be expected to reside preferentially at either nitrogen or sulfur atom. In this context, the electronic charges of different atoms were performed by the molecular modeling and extended Hückel semiempirical calculations using Chem3D Pro 12.0.2 software. To our knowledge, no similar study was reported about the correlation between fragmentation processes in the mass spectrometry of thiosemicarbazone and 4-thiazoline derivatives with electronic charges of their heteroatoms. According to the charges of different atoms in the thiosemicarbazone moiety of compounds 3a-e (Table 1), it is clear that the most probable point of impact of the electron beam is the sulfur atom $\left(\mathrm{S}_{10}\right)$ of the thiocarbonyl $(\mathrm{C}=\mathrm{S})$ group, because it bears the most quantity of negative charge and consequently lowest ionization potential compared to $N_{7}, N_{9}$ and $N_{11}$ atoms. Thus, significant fragmentations giving the most important fragments are derived from the molecular ion obtained by this impact. In all compounds the molecular ions have agreed with the nitrogen rule stated that organic compounds containing exclusively hydrogen, carbon, nitrogen, oxygen, silicon, phosphorus, sulfur and the halogens either have an odd nominal mass that indicates an odd number of nitrogen atoms are present or an even nominal mass that indicates an even number of nitrogen atoms in the molecular formula of the molecular ion.

All the compounds examined yielded easily recognizable molecular ions and the principal fragmentation pathways are illustrated in Scheme 2. The $\mathrm{m} / \mathrm{z}$ ratios of their molecular ions (A) are in good agreement with the calculated exact masses and are an indication of the stability of these ions. In all cases, molecular ion peaks for compounds containing an isotopic halogen appeared with appropriate 
Scheme 2 The pathways for fragmentation of thiosemicarbazones 3a-e<smiles>[NH3+]c1cccc(Cl)c1</smiles>

B

$m / z=127,129$

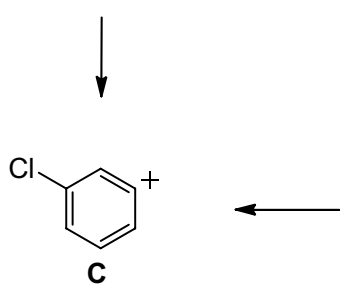

$m / z=111,113$

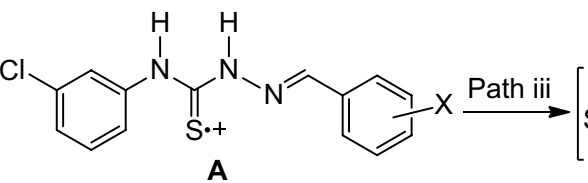

A

3a: $m / z=289,291$

3b: $m / z=334,336$

3c: $m / z=319,321$

3d: $m / z=323,325,327$

3e: $m / z=305,307$<smiles>Fc1cccc([N+](=NC=C=S)c2ccccc2)c1</smiles>

$m / z=169,171$<smiles>[X]c1ccc(/C=N/N=C=S)cc1</smiles>
G

3a: $m / z=162$

3b: $m / z=207$

3c: $m / z=192$

3d: $m / z=196,198$

3e: $m / z=178$<smiles>[X]c1cccc(C=[N+]=[N-])c1</smiles>

H

3a: $m / z=119$

3b: $m / z=164$

3c: $m / z=149$

3d: $m / z=153,155$

3e: $m / z=135$

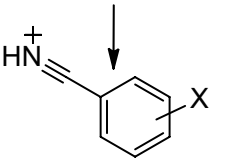

I

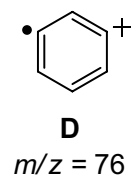

$=76$

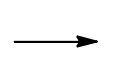<smiles>Fc1ccccc1</smiles>

$m / z=75$<smiles>c1ccccc1</smiles>
$\mathrm{m} / \mathrm{z}=76$<smiles>CCC</smiles>

3a: $m / z=77$

3b: $m / z=122$

3c: $m / z=107$

3d: $m / z=111,113$

3e: $m / z=93$ 3a: $m / z=104$

3b: $m / z=149$

3c: $m / z=134$

3d: $m / z=138,140$

3e: $m / z=120$

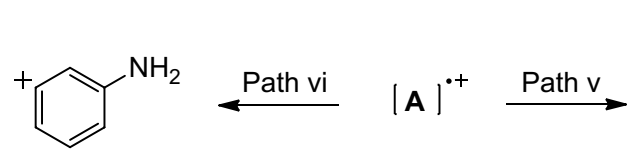

$\mathbf{L}$

$m / z=92$<smiles>[NH3+]C(=S)Nc1cccc(Cl)c1</smiles>

$\mathrm{K}$

$m / z=185,187$

Table 2 Electronic charges of $\mathrm{N}_{1}, \mathrm{~S}_{3}, \mathrm{~N}_{12}, \mathrm{~N}_{13}, \mathrm{C}_{2}$ and $\mathrm{C}_{14}$ atoms of compounds 5 a-e<smiles>[X]c1ccc(/C=N\N=c2/scc(-c3ccc(Br)cc3)n2-c2cccc(Cl)c2)cc1</smiles>

\begin{tabular}{llllrrrr}
\hline Compound & $\mathrm{X}$ & $\mathrm{N}_{1}$ & $\mathrm{~S}_{3}$ & $\mathrm{~N}_{12}$ & $\mathrm{~N}_{13}$ & $\mathrm{C}_{2}$ & $\mathrm{C}_{14}^{\mathrm{a}}$ \\
\hline $\mathbf{5 a}$ & $\mathrm{H}$ & 0.470 & 0.405 & -0.107 & -0.051 & 0.170 & -0.087 \\
$\mathbf{5 b}$ & $4-\mathrm{NO}_{2}$ & 0.440 & 0.416 & 0.569 & 0.602 & 0.171 & 0.018 \\
$\mathbf{5 c}$ & $4-\mathrm{OCH}_{3}$ & 0.463 & 0.389 & -0.166 & -0.079 & 0.178 & -0.083 \\
$\mathbf{5 d}$ & $2-\mathrm{Cl}$ & 0.422 & 0.347 & 0.211 & 0.630 & -0.012 & -0.132 \\
$\mathbf{5 e}$ & $2-\mathrm{OH}$ & 0.465 & 0.397 & -0.154 & 0.113 & 0.178 & -0.074 \\
\hline
\end{tabular}

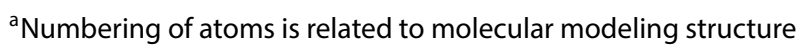


peak intensities. The molecular ions underwent fragmentation in four main pathways (i-iv) to produce a number of reasonable ion peaks (B-J) as a result of cleavage around the $\mathrm{C}=\mathrm{S}$ bond.

All the compounds showed an intense peak at $m / z=127 / 129$ with a relative intensity in a ratio of $3: 1$, corresponding to the molecular formula $\mathrm{C}_{6} \mathrm{H}_{6} \mathrm{NCl}$ (ion B) as shown in path $\mathbf{i}$. This ion constituted the base peak and was identified as 3-chloroaniline ion. It lost $\cdot \mathrm{NH}_{2}$ to give the ion of chlorobenzene (ion $\mathbf{C}$ ) at $m / z=111 / 113$. As a consequence, the fragmentation via a-cleavage process between the $\mathrm{C}=\mathrm{S}$ group and $\mathrm{N}_{7}$ atom of thiosemicarbazone moiety is considered as the most probable one. Thus, a common feature in these mass spectra is the high abundance of the 3-chloroaniline ion, because it is a particularly stable ion. Further fragmentation of ion $\mathbf{C}$ led to a relatively stable aromatic ions $\left[\mathrm{C}_{6} \mathrm{H}_{4}\right]^{+}$(ion $\mathbf{D}$ ) and $\left[\mathrm{C}_{6} \mathrm{H}_{3}\right]^{++}$(ion E) located at $m / z=76$ and 75 , respectively. Also, these ions could be generated from 1-chloro-3-isothiocyanatobenzene ion (ion $\mathbf{F}, \mathrm{C}_{7} \mathrm{H}_{4} \mathrm{CINS}$ ), that produced from molecular ion (A) by the fission of bond between the $\mathrm{C}=\mathrm{S}$ group and
$\mathrm{N}_{9}$ atom (path ii). Other well identified ions (G-J) were obtained via pathways iii and iv. Furthermore, the molecular ions of compounds $\mathbf{3 a - e}$ were also found to undergo fragmentation to produce the ion of 1-(3-chlorophenyl) thiourea (ion K) at $m / z=185 / 187$ (path $\mathbf{v}$ ). The later ion resulted from the cleavage between $\mathrm{N}_{9}$ and $\mathrm{N}_{11}$ atoms, and it may be affected by the inductive and the conjugative effect of substituent $X$, that is already present in the benzene ring. Other significant ion was the aniline cation (ion L), which appeared in all compounds at $m / z=92$ (path vi).

The electronic charges of different atoms in 2-hydrazono-2,3-dihdrothiazoles $\mathbf{5 a - e}$ are given in Table 2. It revealed that two main types of cleavage at $\mathrm{C}_{2}=\mathrm{N}_{12}$ and $\mathrm{C}_{14}=\mathrm{N}_{13}$ bonds are highly appreciated.

The mass spectra of compounds $\mathbf{5 a - e}$ are fully consistent with the assigned structures. In all cases, intense molecular ion peaks were observed due to $[\mathrm{M}]^{+},[\mathrm{M}+2]^{+}$ and $[\mathrm{M}+4]^{+}$ions in addition to $[\mathrm{M}+6]^{+}$in case of compound $\mathbf{5 d}$ containing one bromine and two chlorine atoms. The main fragmentation pathways of compounds $\mathbf{5 a - e}$ are shown in Scheme 3.
Scheme 3 The pathways for fragmentation of 2-hydrazono2(3H)-thiazoles (5a-e)

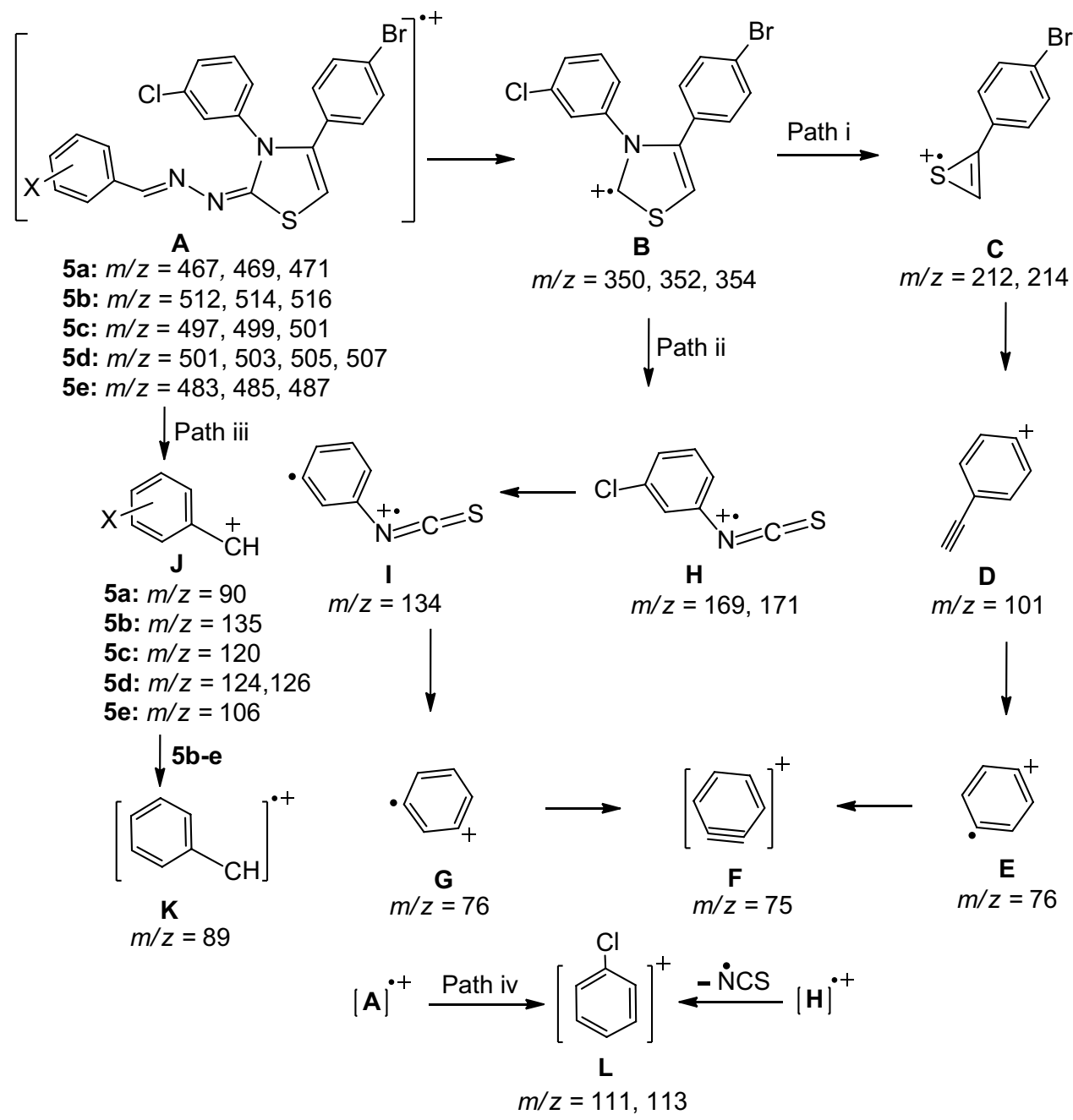


The molecular ion $(\mathbf{A})$ underwent fragmentation at $\mathrm{C}_{2}=\mathrm{N}_{12}$ bond to produce the thiazoline fragment (B) at $m / z=350 / 352 / 354$ as $[M+1]^{+}$peaks, corresponding to the molecular formula $\mathrm{C}_{15} \mathrm{H}_{9} \mathrm{BrCINS}$. It was found to undergo fragmentation in two different pathways (path i, ii) to give the ions of 2-(4-bromophenyl)thiirene (ion C) and 1-chloro-3-isothiocyanatobenzene (ion $\mathbf{H}$ ) at $m / z=212 / 214$ and $169 / 171$, respectively. The abundance of thiirene ion is very low due to its low thermal stability. It thus appears that successive elimination of $\cdot \mathrm{Br}$ and $\mathrm{S}$ is well established to give $\left[\mathrm{C}_{6} \mathrm{H}_{4} \mathrm{C} \equiv \mathrm{CH}\right]^{+}$(ion $\mathbf{D}$ ) at $m / z=101$. Further fragmentations of ions $\mathbf{D}$ and $\mathbf{H}$ led to $\left[\mathrm{C}_{6} \mathrm{H}_{4}\right]^{+}$ (ions $\mathbf{E}$ and $\mathbf{G}$ ) at $m / z=76$, which eliminated $H^{\prime}$ to give $\left[\mathrm{C}_{6} \mathrm{H}_{3}\right]^{+}$(ion $\mathbf{F}$ ) at $m / z=75$. Other significant fragmentation pathway of the molecular ion (path iii) has occurred at $\mathrm{N}_{13}=\mathrm{C}_{14}$ bond to afford the benzal cation (ion $\mathrm{J}$ ) with the formula $\left[\mathrm{XC}_{6} \mathrm{H}_{4} \mathrm{CH}\right]^{+}$. Its high abundance in compounds $\mathbf{5 a}$ $(\mathrm{m} / \mathrm{z}=90,97.38 \%)$ and $\mathbf{5 c}(\mathrm{m} / \mathrm{z}=120,71.15 \%)$ reflected its particular stability. It lost $X$ to give the un-substituted benzal cation (ion $\mathbf{K}$ ) located at $m / z=89$. It was also observed that the molecular ions of compounds $\mathbf{5 a}, \mathbf{5 b}, \mathbf{5 c}$ and $\mathbf{5 e}$ are very stable and present generally an important relative abundance. It is surprising that they have been obtained as the base peaks. However, the 4-thiazoline $\mathbf{5} \mathbf{d}$ gave the benzal ion (K) at $m / z=89$ as base peak. Finally, the molecular ion (A) could undergo fragmentation at the $N_{1}$ of the 4-thiazoline ring (path iv) to produce the chlorobenzene cation (ion $\mathbf{L}$ ) at $m / z=111,113$. The later ion could be also obtained from 1-chloro-3-isothiocyanatobenzene (ion $\mathbf{H}$ ) by the loss of $\cdot$ NCS.

\section{Conclusion}

Several 2-(arylidenehydrazono)-4-(4-bromophenyl)-3-(3chlorophenyl)-2,3-dihydrothiazoles were synthesized by reacting $\mathrm{N}$-(3-chlorophenyl)hydrazinecarbothioamide with different aromatic aldehydes, followed by cyclization with 2-bromo-1-(4-bromophenyl)ethanone in a second step. The synthesized compounds were characterized by spectroscopic methods as well as mass spectrometry. The molecular modeling and atomic charges of heteroatoms in all compounds were performed using Chem3D Pro 12.0.2 software and provided an excellent tool to investigate the fragmentation pathways. The nitrogen rule was also found to be good predictors of molecular ions and their fragmentation patterns.

\section{Compliance with ethical standards}

Conflict of interest The author declare that there is no conflict of interest.

\section{References}

1. Beraldo H, Gambinob D (2004) The wide pharmacological versatility of semicarbazones, thiosemicarbazones and their metal complexes. Mini Rev Med Chem 4(1):31-39. https://doi. org/10.2174/1389557043487484

2. Kalinowski DS, Quach P, Richardson DR (2009) Thiosemicarbazones: the new wave in cancer treatment. Future Med Chem 1(6):1143-1155. https://doi.org/10.4155/fmc.09.80

3. Tenório RP, Góes AJS (2005) Tiossemicarbazonas: Métodos De Obtenção, Aplicações Sintéticas e Importância Biológica. Quim Nova 28(6):1030-1037. https://doi.org/10.1590/S0100-40422 005000600018

4. Suvarapu LN, Somala AR, Kodur JR, Baek RO, Ammireddy VRA (2012) Critical review on analytical and biological applications of thio- and phenylthiosemicarbazones. Asian J Chem 24(5):1889-1898

5. Finch RA, Liu M, Grill SP, Rose WC, Loomis R, Vasquez KM, Cheng Y, Sartorelli AC (2000) Triapine (3-aminopyridine-2-carboxaldehydethiosemicarbazone): a potent inhibitor of ribonucleotide reductase activity with broad spectrum antitumor activity. Biochem Pharmacol 59(8):983-991. https://doi.org/10.1016/S0006 -2952(99)00419-0

6. Quenelle DC, Keith KA, Kern ER (2006) In vitro and in vivo evaluation of isatin-beta thiosemicarbazone and marboran against vaccinia and cowpox virus infections. Antiviral Res 71(1):24-30. https://doi.org/10.1016/j.antiviral.2006.02.010

7. Davidson PT, Le HQ (1992) Drug treatment of tuberculosis-1992. Drugs 43(5):651-673. https://doi.org/10.2165/00003 495-199243050-00003

8. Alam MS, Ahmed JU, Lee D (2014) Synthesis, antibacterial, antioxidant activity and QSAR studies of novel 2-arylidenehydrazinyl-4-arylthiazole analogues. Chem Pharm Bull 62(12):12591268. https://doi.org/10.1248/cpb.c14-00616

9. Carradori S, Bizzarri B, D'Ascenzio M, De Monte C, Grande R, Rivanera D, Zicari A, Mari E, Sabatino M, Patsilinakos A, Rango R, Secci D (2017) Synthesis, biological evaluation and quantitative structure-active relationships of 1,3-thiazolidin-4-one derivatives. A promising chemical scaffold endowed with high antifungal potency and low cytotoxicity. Eur J Med Chem 140:274-292. https://doi.org/10.1016/j.ejmech.2017.09.026026

10. de Aquino TM, Liesen AP, da Silva RE, Lima VT, Carvalho CS, de Faria AR, de Araujo JM, de Lima JG, Alves AJ, de Melo EJT, Goes AJS (2008) Synthesis, anti-Toxoplasma gondii and antimicrobial activities of benzaldehyde 4-phenyl-3-thiosemicarbazones and 2-[(phenylmethylene)hydrazono]-4-oxo-3-phenyl-5-thiazolidine acetic acids. Bioorg Med Chem 16(1):446-456. https://doi. org/10.1016/j.bmc.2007.09.025

11. Ebrahimi HP, Hadi JS, Alsalim TA, Ghali TS, Bolandnazar Z (2015) A novel series of thiosemicarbazone drugs: from synthesis to structure. Spectrochim Acta A Mol Biomol Spectrosc 137:10671077. https://doi.org/10.1016/j.saa.2014.08.146

12. Er M, Şahin A, Tahtaci $H$ (2014) Synthesis and characterization of novel 1,3-thiazole and 2-amino-1,3,4-thiadiazole derivatives. Maced J Chem Chem Eng 33(2):189-198. https://doi. org/10.20450/mjcce.2014.522

13. Gaffer HE, Khalifa ME (2015) Eco-friendly synthesis of some thiosemicarbazones and their applications as intermediates for 5-arylazothiazole disperse dyes. Molecules 20(12):21982-21991. https://doi.org/10.3390/molecules201219820

14. Grozav A, Găină LI, Pileczki V, Crisan O, Silaghi-Dumitrescu L, Therrien B, Zaharia V, Berindan-Neagoe I (2014) The synthesis and antiproliferative activities of new arylidene-hydrazinylthiazole derivatives. Int J Mol Sci 15(12):22059-22072. https:// doi.org/10.3390/ijms151222059 
15. Hassan AA, Shawky AM, Shehatta HS (2012) Chemistry and heterocyclization of thiosemicarbazones. J Heterocycl Chem 49(1):21-37. https://doi.org/10.1002/jhet.677

16. Meo PL, Gruttadauria M, Noto R (2005) Oxidative cyclization of aldehyde thiosemi carbazones induced by potassium ferricyanide and by tris( $p$-bromophenyl)aminohexachloroantimoniate. a joint experimental and computational study. ARKIVOC (i):114-129. https://doi.org/10.3998/ark.5550190.0006.112

17. Metwally MA, Bondock S, El-Azap H, Kandeel EM (2011) Thiosemicarbazides: synthesis and reactions. J Sulfur Chem 32(5):489-519. https://doi.org/10.1080/17415993.2011.601869

18. Noto R, Buccheri F, Cusmano G, Gruttadauria M, Werber G (1991) Substituent effect on oxidative cyclization of aldehyde thiosemicarbazones with ferric chloride. J Heterocycl Chem 28(5):14211427. https://doi.org/10.1002/jhet.5570280539

19. Penta S, Vedula RR (2012) A facile one-pot synthesis of thiazoles and thiazolyl-pyrazole derivatives via multicomponent approach. Org Commun 5(3):143-149

20. Rahim F, Zaman K, Ullah H, Taha M, Wadood A, Javed MT, Rehman W, Ashraf M, Uddin R, Uddin I, Asghar H, Khan A, Khan KM (2015) Synthesis of 4-thiazolidinone analogs as potent in vitro anti-urease agents. Bioorg Chem 63:123-131. https:// doi.org/10.1016/j.bioorg.2015.10.005

21. Shegal LM, Shegal IL (1970) Oxidative cyclization of 4-arylthiosemicarbazones. Chem Heterocycl Compd 6(10):1238-1240. https://doi.org/10.1007/BF00755065

22. Shih MH, Ke FY (2004) Syntheses and evaluation of antioxidant activity of sydnonyl substituted thiazolidinone and thiazoline derivatives. Bioorg Med Chem 12(17):4633-4643. https://doi. org/10.1016/j.bmc.2004.06.033

23. Davyt D, Serra G (2010) Thiazole and oxazole alkaloids: isolation and synthesis. Mar Drugs 8(11):2755-2780. https://doi. org/10.3390/md8112755

24. Gaumont A-C, Gulea M, Levillain J (2009) Overview of the chemistry of 2-thiazolines. Chem Rev 109(3):1371-1401. https://doi. org/10.1021/cr800189z

25. Diness F, Nielsen DS, Fairlie DP (2011) Synthesis of the thiazole-thiazoline fragment of largazole analogues. J Org Chem 76(23):9845-9851. https://doi.org/10.1021/jo201675r

26. Liu J-C, Liang Y, He H-W (2013) Synthesis, characterization and herbicidal activity of new thiazoline derivatives. Chin Chem Lett 24(3):233-235. https://doi.org/10.1016/j.cclet.2013.01.036

27. Hussein MA, Kafafy AN, Abdel-Moty SG, Abou-Ghadir OM (2009) Synthesis and biological activities of new substituted thiazolinequinoline derivatives. Acta Pharm 59(4):365-382. https://doi. org/10.2478/v10007-009-0033-8

28. Abdel Hafez NA, Elsayed MA, El-Shahawi MM, Awad GEA, Alia KA (2018) Synthesis and Antimicrobial Activity of New
Thiazolidine-Based Heterocycles as Rhodanine Analogues. J Heterocycl Chem 55(3):685-691. https://doi.org/10.1002/ jhet.3087

29. Ahmad A, Ahmad A, Sudhakar R, Varshney H, Subbarao N, Ansari S, Rauf Abdul, Khan AU (2017) Designing, synthesis, and antimicrobial action of oxazoline and thiazoline derivatives of fatty acid esters. J Biomol Struct Dyn 35(15):3412-3431. https://doi. org/10.1080/07391102.2016.1255260

30. Wang W, Zhao B, Xu C, Wu W (2012) Synthesis and Antitumor Activity of the Thiazoline and Thiazine Multithioether. Int J Org Chem 2(2):117-120. https://doi.org/10.4236/ijoc.2012.22018

31. Turan-Zitouni G, Leyla Yurttas L, Tabbi A, Çiftçi GA, Temel HE, Kaplancıklı ZA (2018) New Thiazoline-Tetralin Derivatives and Biological Activity Evaluation. Molecules 23(1):E135. https://doi. org/10.3390/molecules23010135

32. Brard L, Singh RK, Lange TS, Kim KK, Singh AP, Hopson R, Vorsa N (2008) Synthesis of Bicyclic Aryl Thiazolines with Selective AntiProliferative Effects on Human Cancer Cell Lines. Lett Org Chem 5(2):103-109. https://doi.org/10.2174/157017808783743966

33. Ramadan E (2016) Synthesis, spectral characterization and antimicrobial activity of novel 2-hydrazono-2(3H)-thiazole derivatives derived from 4-(3-chlorophenyl)-3-thiosemicarbazide. Int J Pharm Pharm Sci 8(7):176-182

34. Ramadan E (2010) Synthesis of Biologically Active [4-(4-Bromophenyl)-2-thiazolyl] hydrazones and their D-Galactose Derivatives. Chin J Chem 28(4):594-600. https://doi. org/10.1002/cjoc.201090118

35. Chattopadhyay D, Banerjee T, Mazumdar SK, Ghosh S, Kuroda R (1991) Structure of 4-(3-chlorophenyl)thiosemicarbazide. Acta Cryst C 47(Part 1):112-115. https://doi.org/10.1107/S010827019 0002852

36. Serra S, Moineaux L, Vancrayenest C, Masereel B, Wouters J, Pochet L, Frédérick R (2014) Thiosemicarbazide, a fragment with promising indolamine-2,3-dioxygenase (IDO) inhibition properties. Eur J Med Chem 82:96-105. https://doi.org/10.1016/j.ejmec h.2014.05.044

37. Huang $H$, Chen $Q$, Ku X, Meng L, Lin L, Wang X, Zhu C, Wang Y, Chen Z, Li M, Jiang H, Chen K, Ding J, Liu H (2010) A series of a-heterocyclic carboxaldehyde thiosemicarbazones inhibit topoisomerase lla catalytic activity. J Med Chem 53(8):30483064. https://doi.org/10.1021/jm9014394

Publisher's Note Springer Nature remains neutral with regard to jurisdictional claims in published maps and institutional affiliations. 